\title{
Imaging in rheumatology: reconciling radiology and rheumatology
}

\author{
Bernhard J. Tins • Robin Butler
}

Received: 25 May 2013 /Revised: 17 September 2013 / Accepted: 19 September 2013 / Published online: 15 October 2013

(C) The Author(s) 2013. This article is published with open access at Springerlink.com

\begin{abstract}
Imaging in rheumatology was in the past largely confined to radiographs of the hands and sacroiliac joints (SIJs) helping to establish the diagnosis and then monitoring disease progression. Radiographs are not very sensitive for early inflammation in inflammatory rheumatic disorders and the demand on imaging services was therefore limited. However, over the last 10-15 years new drugs and new technologies have brought new challenges and opportunities to rheumatology and radiology as specialties. New drug treatments allow more effective treatment, preventing many complications. Early diagnosis and disease monitoring has become the challenge for the rheumatologist and radiologist alike. The best possible patient outcome is only achieved if the two specialties understand each other's viewpoint. This article reviews the role of imaging - in particular radiography, magnet resonance imaging, computer tomography, ultrasound and nuclear medicine - for the diagnosis and monitoring of rheumatological disorders, concentrating on rheumatoid arthritis, inflammatory spondylarthropathies and gout.

Teaching Points

- New drugs for the treatment of inflammatory disorders has led to greatly improved outcomes.

- Imaging often allows for earlier diagnosis of inflammatory disorders.

- Early diagnosis and treatment can often prevent the development of crippling disease manifestations.

- Tailored imaging examinations are best achieved by consultation of rheumatologist and radiologist.
\end{abstract}

\footnotetext{
B. J. Tins $\cdot$ R. Butler Twmpath Lane, Oswestry SY10 7AG, UK

B. J. Tins $(\bowtie)$

Department of Radiology, Oswestry, UK

e-mail: Bernhard.Tins@rjah.nhs.uk

R. Butler $(\bowtie)$

Department of Rheumatology, Oswestry, UK

e-mail: Robin.Butler@rjah.nhs.uk
}

Robert Jones and Agnes Hunt Orthopaedic Hospital, NHS Trust,
Keywords Rheumatology $\cdot$ Radiology $\cdot$ Rheumatoid arthritis $\cdot$ Inflammatory spondylarthropathy $\cdot$ Gout

\section{Introduction}

Imaging in rheumatology used to be largely about radiographs of the hands and sacroiliac joints (SIJs) helping to establish the diagnosis and then imaging of complications. Radiographs are not very sensitive for recording change and often rheumatologists did not feel imaging had much to offer in inflammatory rheumatic disorders and the demand on imaging services was therefore limited. However, over the last $10-15$ years new drugs and new technologies have brought new challenges and opportunities to rheumatology and radiology as specialties. This article tries to explain the different perspectives of radiologists and rheumatologists.

Given the width and detail of investigations radiologists can offer, it is surprising how little use some rheumatologists make of the imaging options available and how little some radiologists understand the crucial role imaging can play in determining patient outcome.

This article aims to discuss the relevance of imaging for the rheumatologist, concentrating on rheumatoid arthritis, inflammatory spondylarthropathies and gout.

\section{Recent changes in the drug treatment of rheumatological diseases}

The treatment of rheumatological disorders has undergone a dramatic change in the last 10 years or so. The days of painkillers plus steroid and sub-optimal use of conventional diseasemodifying anti-rheumatic drugs (DMARDs) for most inflammatory disorders are gone. When biological drugs became available, treatment directed at key players involved in the pathogenesis of the disease rather than just symptomatic treatment became possible. Monoclonal antibodies or other biological agents which inhibit tumour necrosis factor $\alpha(\mathrm{TNF} \alpha)$ and 
interleukin 6 (IL-6) or bind to the CD20 antigen on B cells (rituximab) or interfere with activation of $\mathrm{T}$ cells (abatacept) have been proven to significantly improve clinical outcomes.

Following demonstration of the efficacy of TNF inhibitors in rheumatoid arthritis many rheumatologists were surprised when they were also shown to be effective in ankylosing spondylitis and psoriatic arthritis, given the different clinical and radiological features of these disorders and the fact that different immunological pathways appeared to be involved. However, currently it is not possible to predict who will respond favourably to any given drug. Differences are emerging between rheumatoid arthritis and the seronegative spondyloarthropathies. Despite their efficacy in rheumatoid arthritis, neither abatacept [1] nor tocilizumab [2] appears to be effective in ankylosing spondylitis. Such observations underline the importance of precise clinical diagnosis, in which imaging has an important role to play.

\section{What the rheumatologists need and what they don't need}

The earlier treatment is started the more favourable the longterm prognosis. It has become possible to delay or even prevent severe joint inflammation and subsequent destruction $[2,3]$. To enable early treatment early diagnosis becomes paramount. However, inflammatory joint disorders often start gradually and the clinical features can be non-specific. Not so long ago, several years might have passed before a definite diagnosis was made, by which time irreversible joint damage or destruction had usually occurred.

To enable earlier diagnosis, various rheumatological societies have reviewed their diagnostic criteria and incorporated modern imaging methods and modalities into their diagnostic algorithms.

The role of imaging and radiologists is reviewed according to disease entities (Table 1). When assessing different types of inflammatory rheumatic disorders, the rheumatologist usually requests imaging studies to answer one or more of the following questions:

Table 1 Usefulness of the various imaging modalities for the early diagnosis of inflammatory joint disorders

\begin{tabular}{|c|c|c|c|c|}
\hline & $\mathrm{X}$-ray & US & MRI & $\begin{array}{l}\text { Nuclear } \\
\text { medicine }\end{array}$ \\
\hline Rheumatoid arthritis & - & + & + & + \\
\hline $\begin{array}{l}\text { Inflammatory } \\
\text { spondylarthropathy }\end{array}$ & - & +- & ++ & + \\
\hline Gout & +- & +- & +- & - \\
\hline
\end{tabular}

- means not usually useful, +- means may or may not be useful depending on clinical circumstances, + means usually useful, ++ means method of choice
1. Is there evidence of an inflammatory process?

2. If so which anatomical sites are involved?

3. What is the specific diagnosis?

4. How is the disease responding to treatment?

\section{Rheumatoid arthritis}

Rheumatoid arthritis is still a disease of unclear aetiology. It is an autoimmune disease with a genetic predisposition involving many genes and women are more commonly affected than men. What exactly triggers its onset is subject to lively scientific debate, but it seems likely that once the causative abnormal immune process is established in the patient, it becomes self-perpetuating.

Rheumatoid arthritis is largely a synovial based disease with the potential for severe joint destruction, but it often has systemic manifestations. Some authors have suggested that synovitis might be secondary to a more basic disease process first occurring within medullary bone as primary inflammatory infiltrate [4-7], but this is subject to debate and not universally accepted [8]. Intriguingly, Ostendorf and co-workers [6,9] found that bone scintigraphy has demonstrated increased bone turnover in some patients with rheumatoid arthritis without correlating magnet resonance imaging (MRI) findings, while in most patients bone scintigraphic uptake correlated well with MRI changes.

Older classifications of rheumatoid arthritis were insensitive to early rheumatoid arthritis and years could pass between onset of symptoms and definite diagnosis. However studies have shown that the best outcomes are achieved by aggressive treatment within 3-6 months of onset of symptoms [4, 10, 11]. The availability of biologic disease modifying anti-rheumatic drugs has moved the goalposts and enables disease control and joint preservation unknown in the past but only if treatment is started early enough.

Subsequently the diagnostic criteria for rheumatoid arthritis were revised. The 2010 American College of Rheumatology/ European League against Rheumatism (EULAR) classification assesses joint involvement, blood parameters and duration of symptoms. Unlike the previous criteria, imaging is not part of this primary assessment, though radiographs of the hands might be obtained at the time of diagnosis to establish baseline findings [12], although, clearly, radiographs are insensitive for the early diagnosis of inflammatory joint disease. Radiographs can be used to monitor disease progression, although they are insensitive for minor change.

Disease activity and response to treatment are usually assessed by clinical criteria and blood tests. For straightforward cases of rheumatoid arthritis, the rheumatologist might therefore not need much or any imaging input.

Imaging is, however, required for all cases that are not straightforward. Patients presenting with fairly non-specific 
aches, not fulfilling the diagnostic criteria for rheumatoid arthritis or other inflammatory disease, might be early presentations of these disorders, and imaging can help in the differential diagnosis of inflammatory rheumatic disorders. As early diagnosis results in far better long-term outcomes, a wait and see approach is no longer acceptable.

The questions asked of the radiologist in these cases are typically some or all of these.

Is there inflammation? Which joint or organ is affected? What is the anatomical pattern of involvement?

\section{Radiographs}

Radiographs are insensitive to most forms of soft tissue inflammation and usually play little role in these cases $[3,13]$. Radiographs of the hands or other inflamed joints mostly show normal bone structure and alignment in early disease (Fig. 1) and are requested by some rheumatologists to establish a baseline. The earliest radiographic findings or rheumatoid arthritis are periarticular soft tissue swelling, followed by osteopenia and bone erosions. In early disease the joint space may appear widened due to joint effusion and synovitis. In latter stages joint destruction, bone union and malalignment may occur (Fig. 2). The early disease stages, soft tissue swelling, osteopenia and even bone erosions are still reversible. Obviously once cartilage destruction has occurred this is irreversible and, even if the rheumatoid arthritis is halted, secondary osteoarthritis will ensue $[3,4,9,13-15]$.

\section{MRI}

MRI is a very sensitive examination for inflammation be it in soft tissue or bone. Clearly there has to be some understanding of which areas are to be investigated. Whole-body MRI is not well suited for the assessment of rheumatoid arthritis because the small joints in particular are not well imaged. In early disease the use of contrast medium enhancement can increase the sensitivity for mild tenosynovitis, joint synovitis and bone marrow signal change (Fig. 3) [3, 13, 15]. If contrast medium administration is performed as a dynamic examination, the contrast medium enhancement curve can be reviewed and assessed for its enhancement pattern, further increasing the sensitivity and specificity of the examination [16]. To perform a dynamic contrast-enhanced examination the area of interest is repeatedly imaged. Usually the image acquisition begins before the contrast-medium injection or immediately after the injection, before the contrast medium can circulate to the examined area. This way it is possible to subtract the anatomical image information and display the inflow of contrast medium into the area of interest; this allows the speed and intensity of contrast-medium enhancement to be observed, which in turn allows conclusions to be drawn about the target tissues. It might also permit assessment of disease activity. However, in most cases the rheumatologist simply wants to know whether inflammation is present - and, if so, where? and a dynamic examination is not necessary.

Dynamic contrast-medium-enhanced MRI is ideally done by imaging the whole volume of interest, for example with a three-dimensional (3D) sequence. Using a 3D sequence puts higher demands on the scanner hardware but is otherwise no different from using conventional 2D sequences.

Delayed post-contrast-enhancement MRI is not always able to differentiate between synovium and joint fluid because it might only take a few minutes for contrast medium to diffuse into perisynovial fluid, particularly in cases of florid inflammation [17].

MRI in rheumatology imaging can be reported using reference sets and scoring systems. The European league against rheumatism (EULAR) has published reference atlases and viewing instructions [18-20] which are available on the EULAR website (www.eular.org). One of the main reasons for this is that in many countries rheumatologists perform and
Fig. 1 A 45-year-old woman with known rheumatoid arthritis, complaining of recurrence of mild symptoms after reduction of methotrexate medication. Radiographs of the hands were normal (shown here X-ray left hand, a). Ultrasound demonstrates a bone erosion of the second metacarpal head with mild hypervascularity (b), indicating active mild inflammation. Ultrasound is far more sensitive than radiography for inflammatory joint change
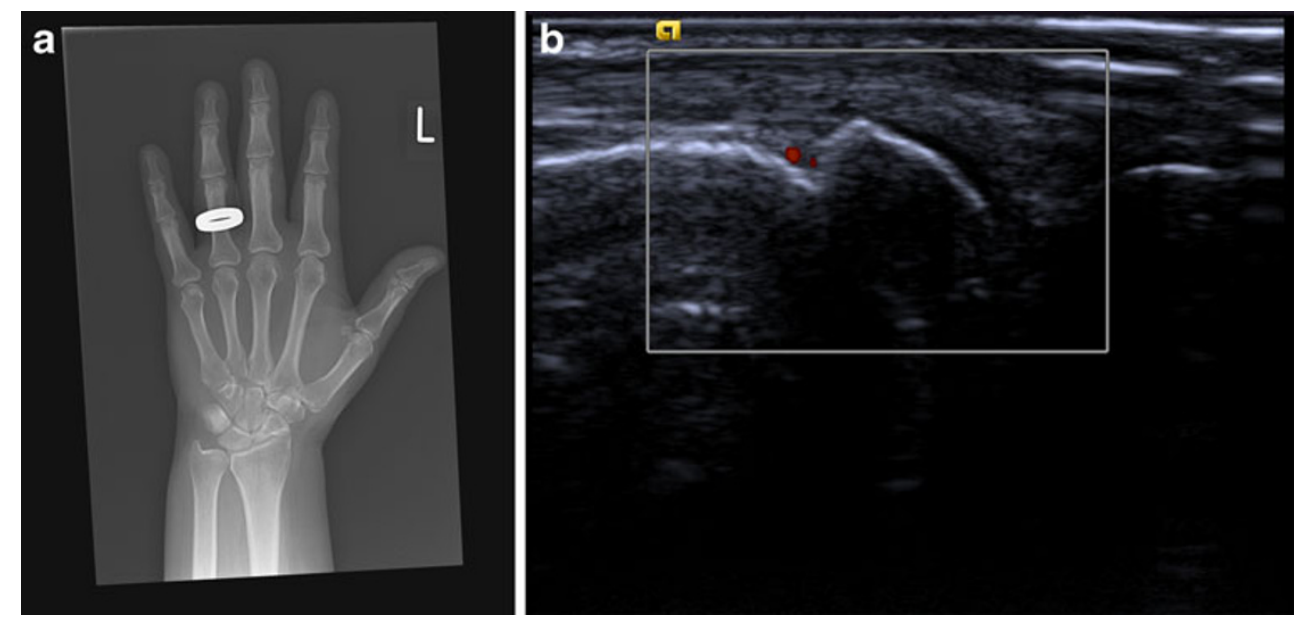
Fig. 2 A 64-year-old woman with severe long-standing rheumatoid arthritis. A radiograph of the left hand shows osteopenia, destruction of the metacarpophalangeal joints (MCPJs) and wrist joint, and fusion of the carpal bones. Outcomes like these should hopefully become extinct with early diagnosis and treatment

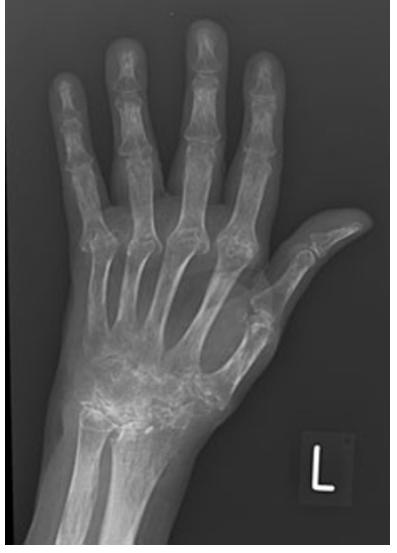

interpret imaging investigations like ultrasound and MRI themselves. It has been shown that rheumatologists largely concentrate on short tau inversion recovery (STIR) images when interpreting MRI and also relying heavily on contrastmedium-enhanced T1-weighted (T1w) images, while relatively ignoring conventional $\mathrm{T} 1 \mathrm{w}$ images [21-23]. This is reflected in the published literature, particularly in the rheumatological literature and can lead to discrepancies in published recommendations.

The outcome measures in rheumatoid arthritis clinical trials (OMERACT) MRI study group recommends that MRI studies should include at least imaging in two planes, T1w imaging before and after intravenous gadolinium based contrast medium, and a fluid-sensitive sequence such as fat saturated T2 (T2 fat sat) or a STIR sequence. If the examination is performed to assess destructive bone changes such as erosions, then i.v. contrast medium enhancement is not mandatory. The image slice thickness should be 3-4 $\mathrm{mm}$ with an interslice gap of $10 \%$ [24]. Any disease process leading to an increased amount of fluid will show increased signal on the fluid sensitive sequences (T2 fat sat and STIR) and decreased signal in T1w images.

Last but not least, MRI might be requested when typical complications of rheumatoid arthritis are suspected such as erosion of the dens axis and transverse ligament of the atlas leading to atlantoaxial instability. Plain films can be difficult to assess in these circumstances and MRI not only demonstrates the amount of erosion and inflammation but can also assess possible neural compromise.

\section{Ultrasound}

Ultrasound is well able to assess superficial joints such as the MCPJs of the hands for joint effusion, synovial proliferation and synovitis as well as bone erosion (Fig. 1) [25]. It is actually not very time-consuming once the small initial learning curve has been overcome. It can assess bursae and larger joints and entheses. However, the more areas investigated the more time-consuming it becomes. Naredo et al. [26] have shown that for routine diagnostic and treatment response purposes it suffices to assess the anterior and posterior recess of the elbow, the dorsal carpal recess of the wrist, the second and third MCPJ dorsally and palmar, the suprapatellar recess
Fig. 3 A 53-year-old man with bilateral inflammation of both hands. Coronal T2 fat saturated (fat sat) MRI (a) shows increased fluid signal consistent with synovitis and oedema in the wrist, intercarpal and metacarpophalangeal joints (MCPJs) and also signal change around the tendons suggesting tenosynovitis. This was confirmed with contrast-mediumenhanced MRI of the hands. Here shown are axial $\mathrm{T} 1$ fat sat images at the level of the MCPJs (b) and the wrist joints (c). Note the erosions in the head of the right second metacarpal (b, arrow)
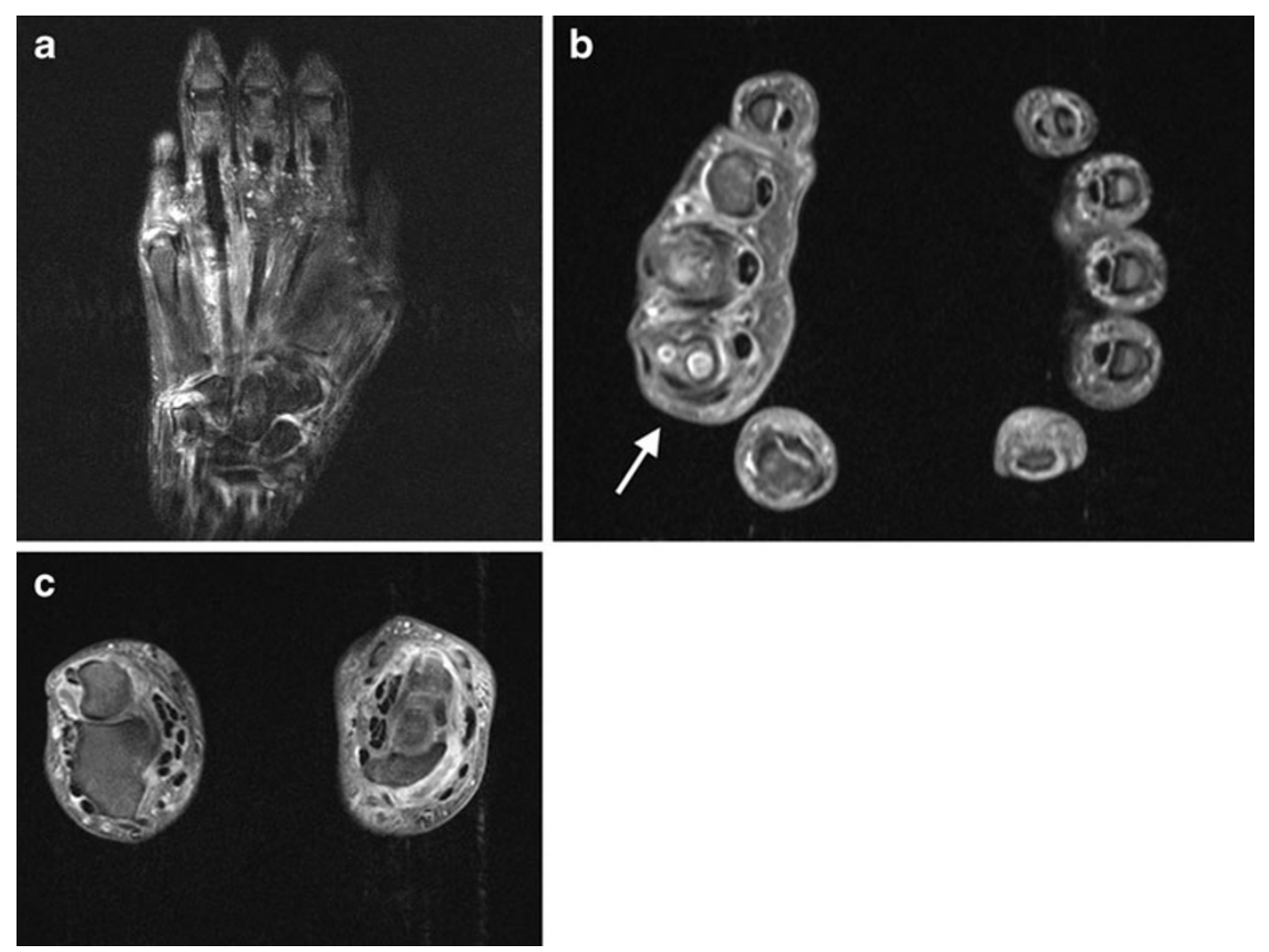
and the lateral parapatellar recess of the knee, the anterior tibiotalar recess of the ankle and the medial and lateral tendon sheaths in the ankle area. In a recent review Spencer et al. [25] suggested more focused concentration on the wrist area and the MCPJs for assessment.

Ultrasound can be combined with the use of contrast medium, but this is not necessary as the most important question for the rheumatologist is usually whether inflammation is present or not, rather than trying to quantify disease activity. Even disease response to treatment can be assessed with a semiquantitative score for synovitis and apart from scientific studies contrast-enhanced ultrasound seems unnecessary [27, 28].

There are many competing assessment and scoring systems and a near constant stream of publications in the rheumatology literature assessing various aspects of this. This might be offputting for radiologists, but the relevance of these is limited for most routine assessments. The rheumatologist knows best which area he or she is interested in and, if indicated, which scoring system is preferred.

When considering whether to use ultrasound or MRI it is worth remembering that ultrasound cannot of course image bone marrow. If this is important then MRI is clearly the modality of choice. Ultrasound is well suited to assess a large number of different joints for inflammation relatively quickly. Whether ultrasound or MRI is more sensitive or specific for synovitis and erosions in a given superficial joint is not yet clear. The comparison is problematic because of the lack of a "gold standard": both modalities have been shown to miss inflammatory soft tissue changes and erosive bone lesions [29, 30]. In larger, deeper joints such as the shoulder, MRI seems more reliable [13].

\section{Nuclear medicine}

Nuclear medicine can and is still being used to assess inflammatory arthropathies. As with all imaging modalities, local preference can vary significantly: availability, expertise, cost and general familiarity all play a role.

In particular, the vascular and diffusion phase can provide information about the presence and degree of inflammation. The bone phase is much less specific and needs correlation with radiographs for interpretation [9, 14]. High-resolution single photon emission computed tomography (SPECT) increases the spatial resolution and allows for better recognition of bone erosions and lesions. Ostendorf et al. [9] even found increased activity in a few patients with normal MRI. It was unclear, though, whether these were patients who would go on to develop rheumatoid arthritis. A study by Ozgul et al. [31] found bone scintigraphy of limited value in predicting or identifying rheumatoid arthritis in patients with early undifferentiated arthritis. Duer et al. [32], however, found the opposite: the three-phase bone scan performed in patients with undifferentiated arthritis was able to predict the presence or absence of rheumatoid arthritis 2 years later quite reliably. It is also able to predict disease progression on later imaging and was found to perform well compared with MRI [33]. More recently a pilot study by Roimicher et al. [34] using Tc99labelled anti-TNF $\alpha$ antibodies showed promising sensitivity (98.8 \%) and specificity (97.3.\%) when compared to MRI in eight patients with rheumatoid arthritis, looking at 198 joints, 49 of which showed inflammation. By contrast clinical assessment only reached a sensitivity of $59 \%$ and specificity of $65 \%$ when compared with MRI.

Overall these studies add to the uncertainty as to whether there is truly an imaging gold standard in inflammatory arthritis. More advanced nuclear medicine techniques do not play a routine role in rheumatology imaging.

In summary, the rheumatologist might not need much radiology in the diagnosis and management of rheumatoid arthritis. If the diagnosis is clear, based on clinical and laboratory parameters, no diagnostic imaging may be required. Baseline radiographs of the hands are usually the only imaging investigation.

If the diagnosis is not clear, imaging might be used to establish the location or nature of inflammation. Which modality is preferred will depend partly on local availability and expertise. For most questions, straightforward MRI—possibly with contrast-medium enhancement - and straightforward ultrasound will suffice. While additional information can be gleaned by quantitative techniques, this is not necessary in routine practice. If a particular scoring system is to be used, this should be indicated by the rheumatologist.

\section{Inflammatory spondyloarthropathies}

Inflammatory spondyloarthropathies consist of a wide range of diseases. The archetypical representative is ankylosing spondylitis, but reactive arthritis (Reiter's syndrome), spondyloarthropathy associated with inflammatory bowel disease, psoriatic arthritis, undifferentiated spondyloarthropathy and, arguably, Whipple's disease and Behcet's syndrome can all be classed as inflammatory spondyloarthropathy. They are linked to the HLA B27 antigen to variable degrees and enthesitis is a key feature [35].

The patient might present with clinical features suggestive of an inflammatory spondyloarthropathy, such as inflammatory back pain, heel pain, peripheral arthritis, dactylitis or similar, and imaging is requested to establish the diagnosis. Differentiation from rheumatoid arthritis in the early stages of the disease when there are limited clinical, laboratory and imaging features can be difficult but establishing the correct diagnosis is important [36].

In other cases the patient might be known to have an inflammatory spondyloarthropathy and imaging is requested 
to narrow the differential diagnosis, e.g. psoriatic spondyloarthropathy versus classical ankylosing spondylitis.

\section{MRI}

The most frequent imaging request in this context is MRI of the SIJs to look for sacroiliitis (Fig. 4). Radiographs can take many years to show evidence of sacroiliitis and the bone scan is unreliable (Fig. 5). Computer tomography (CT) is more sensitive than radiography but cannot reliably show bone marrow change [36, 37].

When requesting an MRI of the SIJs, rheumatologists often request a contrast-medium-enhanced examination. The reason for this is not clear to many radiologists and requests are subsequently either ignored or performed grudgingly. The reason many rheumatologists request a contrast-medium enhanced examination is that this is what is done in many of the studies published in their literature. As discussed in the section on rheumatoid arthritis, many imaging investigations including MRI in countries outside the United Kingdom (UK) are supervised and interpreted by non-radiologists, and they often rely heavily on STIR and contrast-medium-enhanced T1w (often $\mathrm{T} 1$ fat saturation) sequences and do not appreciate the role of conventional $\mathrm{T} 1 \mathrm{w}$ sequences. Radiologists, on the other hand, use both sequences to assess the examined area and usually do not require further sequences.

Most pathological disease processes are associated with an increased amount of free fluid and this is seen as signal increase on fluid-sensitive sequences such as T2 fat sat and STIR and also as signal decrease on conventional $\mathrm{T} 1 \mathrm{w}$ sequences. The anatomical resolution of standard $\mathrm{T} 1 \mathrm{w}$ sequences is usually very high and allows for good anatomical correlation of the findings. The combination of a fat-suppressed fluid-sensitive sequence and a T1w sequence also allows to identify fatty (low signal in fat sat, high in T1w) and sclerotic (low signal in all sequences) bone marrow change.
Compared with STIR sequences, contrast-mediumenhanced $\mathrm{T} 1 \mathrm{w}$ sequences have not been proved to be superior in assessment of spinal inflammation in studies by Baraklios et al. [22] and Madsen et al. [38]. On the other hand, contrastenhanced $\mathrm{T} 1 \mathrm{w}$ studies were found to be slightly superior to STIR imaging in two other studies $[39,40]$.

The importance of training for the reliable diagnosis of inflammatory spondyloarthropathy based on MRI has been stressed [21], and reference instruction manuals and atlases for this purpose have been published [41].

Contrast-medium enhancement and diffusion-weighted imaging with MRI have been found to be more accurate for quantification of treatment response in ankylosing spondylitis [42]. Whether this proves useful in routine clinical imaging remains to be seen.

The use of more exotic contrast medium such as (ultrasmall) superparamagnetic iron oxide particles ([U]SPIO) has also been investigated for the imaging of inflammatory bone marrow lesions. The aim is to define the reason for bone marrow oedema-like change more accurately. As SPIOs are taken up by macrophages, signal change after SPIO administration would indicate the presence of macrophages. This is usually suggestive of infection or severe inflammatory spondyloarthropathy [43]. However this is a research application and unlikely to come into routine practice soon or indeed ever [44-46].

Whether an axial sequence is necessary or whether the paracoronal plane alone is sufficient for imaging of the SIJs is also subject to debate in the relevant literature [47]. Most publications favour the use of the paracoronal plane only and many radiologists are content with this. However, in almost all imaging examinations it is regarded good practice to image in two planes and this reduces uncertainty regarding partial volume effects. The use of 3D sequences in the spine has been found to be useful in this regard [48].

Usually the SIJs are imaged with a dedicated examination focusing on the sacrum and SIJs. However, in clinical practice
Fig. 4 Coronal images of the SIJs demonstrating typical findings of acute or chronic sacroiliitis with sclerosis, fatty and oedema-like change as shown on STIR (a) and T1w (b) images. The diagnosis is not in question
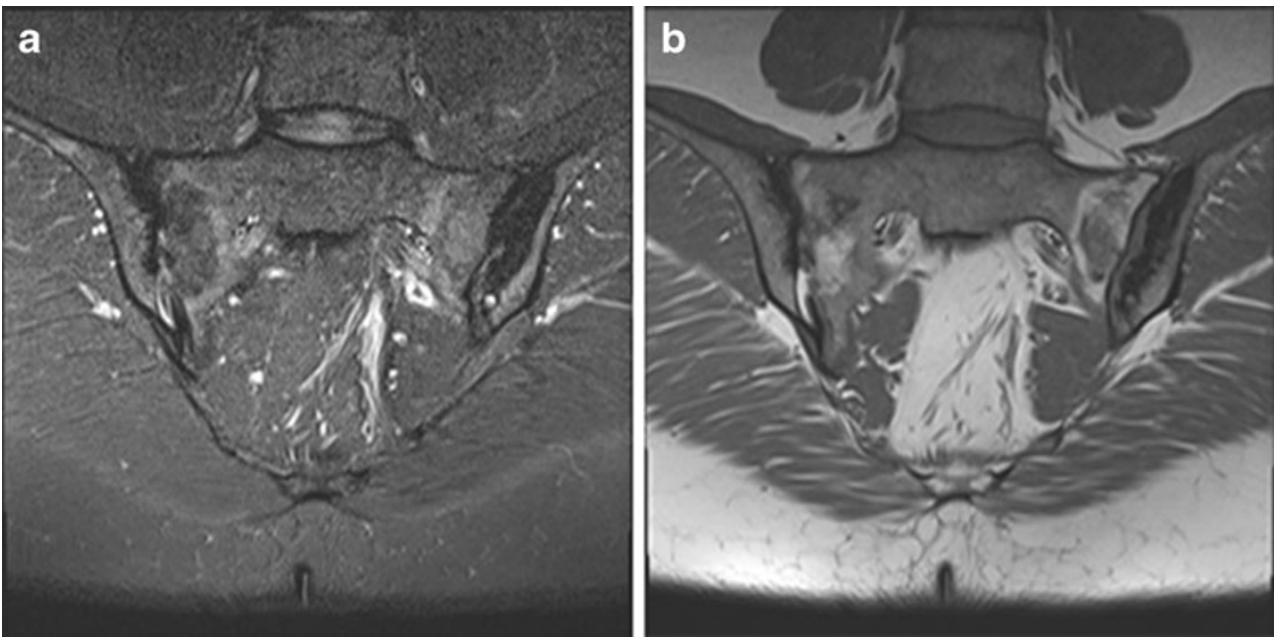

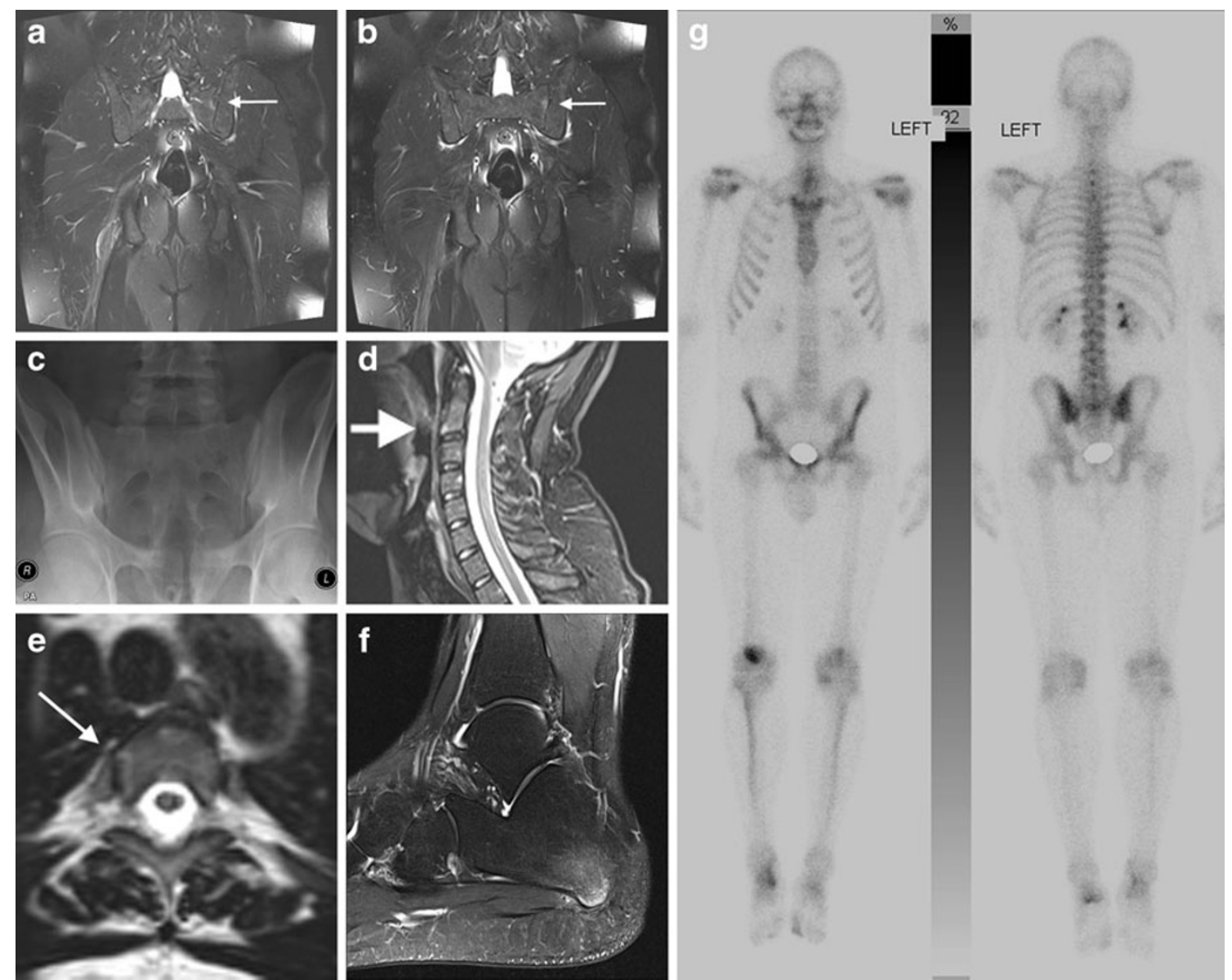

Fig. 5 A 35-year-old man with psoriasis and psoriatic spondylarthropathy. MRI of the pelvis demonstrates a single inflammatory lesion in the left SIJ seen on two adjacent slices, this was best appreciated on the coronal STIR sequence (a, b, arrows). This patient therefore has sacroiliitis. Had the lesion been seen on only one slice, sacroiliitis could not be diagnosed using the ASAS criteria. Sacroiliitis is not visible on the normal-appearing radiograph of the SIJs (c). There is mild involvement of the spine. The sagittal STIR sequence demonstrates mild bone marrow oedema-like change on the anterior aspect of $\mathrm{C} 2$ (d, arrow) consistent with enthesitis.

the SIJs might be imaged as part of an MRI of the pelvis or a whole-body MRI. It has been shown that even imaging with straight coronal sequences with possibly slightly thicker slices allows for reliable diagnosis of sacroiliitis [49].

The diagnostic criteria constituting sacroiliitis have been laid out by the Assessment of Spondyloarthritis International Society (ASAS). They are available online (http://www.asas-group.org) or in print version [41] and are as follows:

Active inflammatory lesions of the SIJs, i.e. bone marrow oedema (as seen on STIR) or osteitis (seen on T1w post contrast-enhanced images), must be clearly present in typical anatomical areas (subchondral or periarticular bone marrow oedema). A single lesion on a single slice is not sufficient for the diagnosis for sacroiliitis. The minimum criteria are two lesions seen on at least one slice each or one lesion seen on two adjacent slices. The presence of synovitis, enthesitis or
The arthropathy is better seen in some costovertebral joints (e, arrow), axial reformat of a sagittally acquired 3D sequence. Volume 3D sequences of the spine enable assessment of all costovertebral and costotransverse joints and can help make the diagnosis. The patient also complained of left foot pain, sagittal T2 fat sat MRI showed enthesitis at the origin of the abductor digiti minimi (f). Bone marrow lesions are not usually visible with ultrasound, $\mathrm{CT}$ or radiographs. Whole body bone scintigraphy demonstrated several areas of abnormal activity but the sacroiliitis and the spine involvement are not appreciable $(\mathbf{g})$ capsulitis on their own are not sufficient to diagnose sacroiliitis on MRI. Structural changes such as fat deposition, sclerosis, erosions or ankylosis are likely to be sequelae of previous inflammation; however, in the absence of acute changes they are not diagnostic of sacroiliitis $[41,50]$.

Interestingly there is no inclusion of imaging parameters such as slice thickness or interslice distance and lesion size is also not part of the assessment. This does in the authors' opinion introduce an element of chance into the assessment. A single lesion of the same size might be visualised on one or two slices depending on its position relative to the centre of the slice, i.e. if it is central it might be seen on one slice only, if it falls onto the border of two slices it might be seen on two slices and therefore fulfil the diagnostic criteria for sacroiliitis. Or putting it another way, the thinner the slices the higher the chance of diagnosing sacroiliitis. 
It is, however, necessary to sound a word of caution regarding the use of MRI to diagnose axial spondyloarthritis according to the ASAS criteria. More patients will meet these criteria than the modified New York criteria, of whom a substantial proportion fail to meet the modified New York criteria at follow-up [51]. The modified New York criteria look for clinical signs of low-back pain improved by exercise but not rest, limitation of lumbar movement and limitation of chest expansion. Further, they assess for radiographic evidence of sacroiliitis of either at least minimal sacroiliitis bilaterally or at least moderate sacroiliitis unilaterally. If either radiographic condition is fulfilled and there is at least one clinical sign, then ankylosing spondylitis is diagnosed.

People with axial spondyloarthropathy are a more heterogeneous group and have a higher proportion of women and more marked genetic differences compared with people with classical ankylosing spondylitis.

There are a number of scoring systems for the assessment of ankylosing spondylitis [52]. As discussed in the section on imaging of rheumatoid arthritis, if this is desired the rheumatologist should indicate which system is preferred.

Similarly there are scoring systems for other inflammatory spondylarthropathies such as psoriasis, for example the OMERACT Psoriatic Arthritis Magnetic Resonance Imaging Score (PsAMRIS) [53]. Should the use of a particular scoring system be of interest, the rheumatologist should indicate this.

\section{Radiographs and CT}

Radiographs might show evidence of bone erosion and/or other reactive bone change with ankylosis of the spine the most extreme form of reactive bone change. While it is, of course, important to note these findings and suggest the diagnosis if unsuspected, radiographs are of limited value in the diagnosis of early inflammatory spondyloarthropathy because of the high false-negative rate (Fig. 5). To an even greater extent than in rheumatoid arthritis, the radiographic changes often lag behind the clinical presentation by years $[4,21,37]$. Nevertheless, rheumatologists in the UK often request plain $\mathrm{X}$-rays because the current UK NICE (National Institute for Health and Care excellence) criteria for the use of TNFinhibitors in ankylosing spondylitis require that the patient fulfils the modified New York radiographic criteria [54].

While CT is more sensitive for reactive bone change it is insensitive for oedema-like change of bone marrow or soft tissues and MRI is preferred for this [36, 37].

\section{Nuclear medicine}

Bone scintigraphy in particular has been used in the past to seek sacroiliitis. Activity ratios of the SIJs versus normal-appearing bone areas can be calculated, but a literature review by Song et al. [55] found the sensitivity with this method to be just over $50 \%$ and therefore quite poor (Fig. 5). It has been suggested that bone scintigraphy be combined with bone marrow scintigraphy but doubts regarding the usefulness of this technique remain [56].

One advantage of bone scintigraphy is that it is by its nature a whole-body imaging technique and hence useful for identification of inflammatory lesions in joints and entheses which are not necessarily apparent clinically. The pattern of involvement can give important diagnostic clues or might detect hitherto unrecognised areas of disease involvement. The combination with single photon emission computer tomography (SPECT) increases anatomical resolution. Whole-body bone scintigraphy might also help differentiate inflammatory spondyloarthropathies from other inflammatory disorders such as SAPHO (synovitis, acne, pustulosis, hyperostosis, osteitis), e.g. by demonstrating the pathognomonic "bull's head" appearance of SAPHO (Fig. 6) [57, 58].

Positron emission tomography (PET)-CT can be used for the diagnosis of inflammatory lesions such as sacroiliitis [59] but the limited availability, cost and radiation dose restrict this technique to scientific interest only for the time being.

\section{Ultrasound}

While ultrasound is, of course, not suited to assess bone marrow disease or spinal arthropathy, it is well suited to look for peripheral enthesitis and can be surprisingly useful in inflammatory spondyloarthropathies [60]. It has even been applied to the diagnosis of sacroiliitis as a low tech alternative to MRI [61]. Orientating ultrasound assessment of major entheses has been suggested in suspected or established cases of inflammatory spondylarthropathies, either to support or refute the diagnosis or to help establish the disease burden [62-64]. D'Agostino et al. [64] assessed about 200 patients with an inflammatory spondyloarthropathy or rheumatoid arthritis and more than 60 controls. They sonographically examined the major entheses of the limbs, the greater trochanters, pubis, patellae, Achilles tendon insertion, planta fascia origin, tibialis anterior insertions, medial and lateral epicondyles. This took about $20 \mathrm{~min}$ and identified more than double the number of areas of enthesopathy as were apparent clinically in the inflammatory spondyloarthropathy group. The pattern of inflammation of entheses or joints can sometimes help to distinguish rheumatoid arthritis from inflammatory spondyloarthropathies and might be requested for this reason. The sonographic findings as such cannot differentiate the various inflammatory spondylarthropathies.

Tinazzi et al. [65] suggested that ultrasound can help to predict the likelihood of developing psoriatic arthropathy in patients with psoriasis based on sonographically visible but 
Fig. 6 A 57-year-old woman with sternoclavicular joint pain. MRI showed bone marrow oedema-like change and synovitis in relation to the sternoclavicular joints and less marked in relation to the corpomanubrial joint of the sternum (a, coronal STIR). Bone scintigraphy demonstrated increased activity in this area, the so-called Bull's head sign (b).

The patient was diagnosed with SAPHO

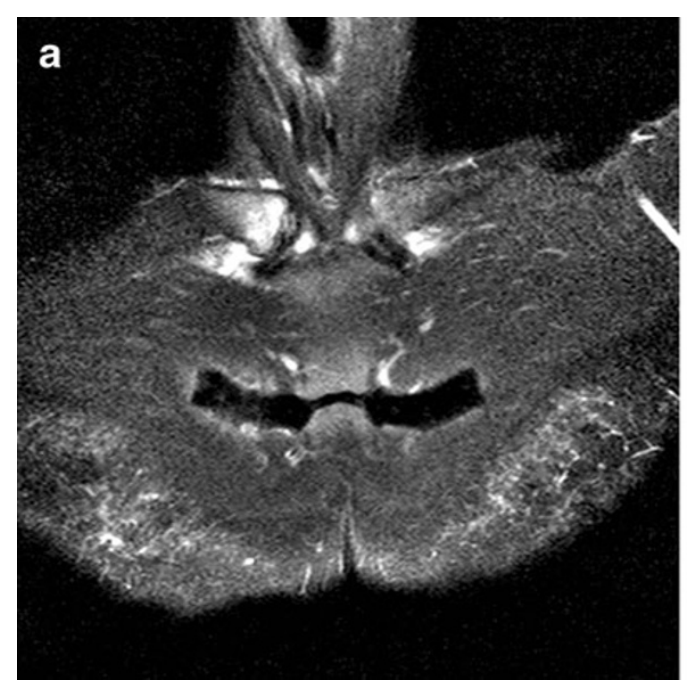

b

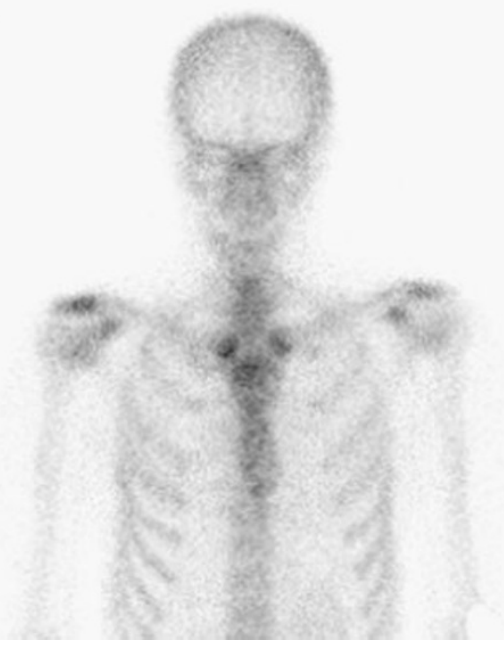

clinically asymptomatic tendon changes. Ultrasound can also be used to assess treatment response and shows decreased inflammation, decrease tendon and enthesis thickness and increase in echogenicity under successful therapy [66].

In conclusion, imaging in inflammatory spondyloarthropathies extends beyond assessment of sacroiliitis, although this examination is probably most frequently requested, and early diagnosis and treatment with TNF-inhibitors can result in a profound improvement in long-term morbidity. There are still requests for radiographs of the SIJs for suspected sacroiliitis and there is an onus on radiologists to publicise the advantages of MRI for early diagnosis. In addition to sacroiliac imaging focal joint or spine imaging can help to assess disease burden and differential diagnosis and can be used to guide steroid injections.
Fig. 7 A 73-year-old man with anterior knee pain and soft tissue swelling suspicious for bursitis. Ultrasound demonstrated a highly echogenic prepatellar mass, not typical for bursitis (a, sagittal extended field of view image). MRI in T1w (b, sagittal) and T2w (c, axial) showed a lowish signal $\mathrm{T} 1$ and mixed signal $\mathrm{T} 2$ lesion. The appearances are unspecific. Biopsy confirmed a gout tophus. Gout tophi can have varied appearances on MRI, the diagnosis in atypical locations in difficult based on imaging alone
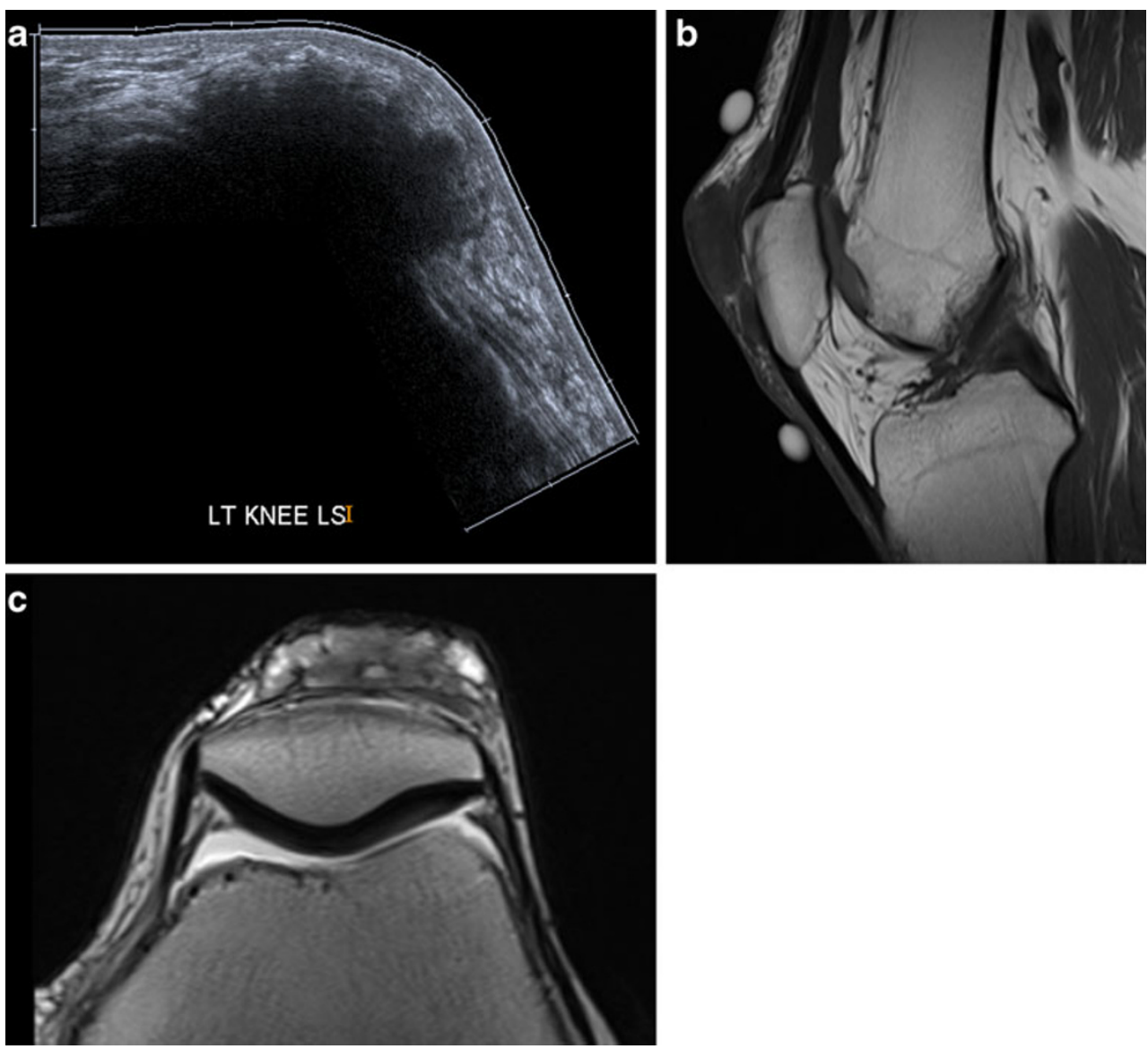

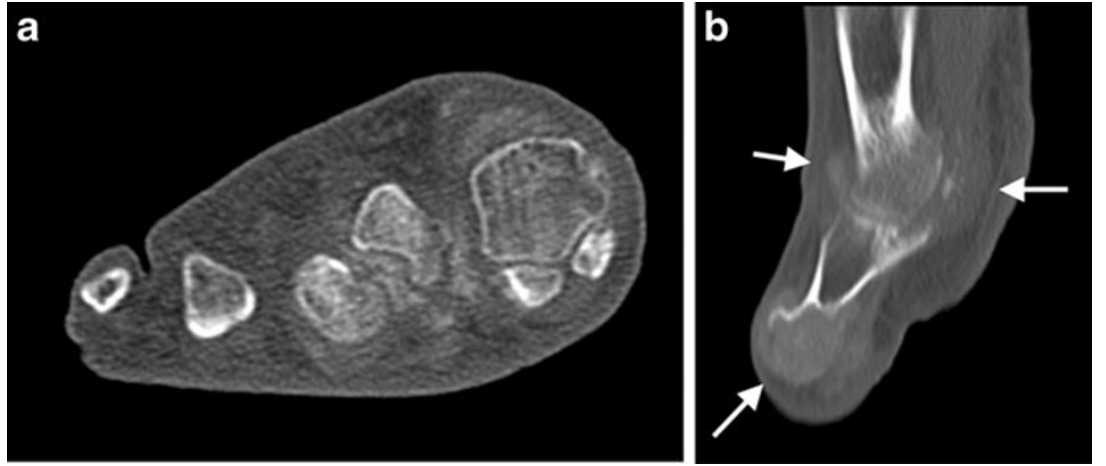

Fig. 8 An 82-year-old man with pain and inflammation in the forefoot. CT demonstrates soft tissue (a coronal reformat of the forefoot, sagittal reformat of the big toe) and destructive bone and articular (b, arrows) deposits, hyperattenuating compared with muscle and hypoattenuating

\section{Gout}

Imaging had a limited role to play in gout until recently because soft tissue opacification and bone lesions do not appear until the later stages of the disease, by which time the diagnosis is usually obvious clinically.

However, the clinical presentation and history of gout are not always typical; the serum urate can drop within the normal range during an acute attack and the "gold standard" for diagnosis, i.e. identification of urate crystals in joint fluid, is frequently not practicable.

Gouty lesions can, however, be identified readily by ultrasound and MRI, which offer scope for improvement in the management of the condition.

Ultrasound can be used to demonstrate tophi and synovial changes, and demonstrates most of the lesions seen on MRI. A double-contour sign (a hyperechoic irregular band over the articular cartilage best seen on the dorsal side of metatarsophalangeal joints) is considered to be pathognomonic. It is thought to represent a layer of urate crystals over the cartilage [67]. However, tophi are sonographically unspecific in appearance (Fig. 7). Compared with MRI, ultrasound is less sensitive as regards to cortical erosions, but better than radiographs. Although changes can be detected over time, it remains unclear whether serial assessment of tophi, synovial change or erosions will prove most useful in the management of gout.

MRI can also be used to demonstrate tophi, synovial changes and erosions. Although less operator-dependent than ultrasound, the lower cost and greater accessibility of the latter are likely to make it the more popular modality for the imaging of gout [68].

The MRI findings of gout obviously depend on the clinical presentation/stage of the disease. Once a tophus has developed, this is visible on MRI imaging, but unfortunately the findings are not pathognomonic. In T1w a tophus is typically isointense to muscle and in $\mathrm{T} 2 \mathrm{w}$ the signal intensity can vary between hypointense and hyperintense, with an intermediate signal intensity most common. This can cause diagnostic compared with bone. The attenuation values were around $200 \mathrm{HU}$ confirming the clinical diagnosis of gout. Masses with attenuation of 150-200 HU are usually due to gout tophi

difficulties and if a tophus is seen in an atypical location even a malignant soft tissue mass may be considered. Other crystal arthropathies, such as calcium pyrophosphate deposition disease, and disorders, such as rheumatoid arthritis, may also have similar imaging findings. Contrast-medium-enhancement is often seen [69]. The best discriminators are typical location and clinical presentation, the presence of typical bone erosions is also helpful for the diagnosis. There is no special imaging protocol showing pathognomonic findings.

CT imaging can be helpful, gout tophi typically demonstrate attenuation values of 150-200 Hounsfield units (HU) and this can be diagnostically helpful (Fig. 8). More recently, dual-energy CT has been found to be more specific in identifying gouty deposits [70, 71].

Earlier diagnosis by either of these methods should accelerate definitive treatment with urate-lowering treatment such as allopurinol or febuxostat, not least because the demonstration of an established burden of crystalline deposits will help to persuade patients to start such treatment. Modern treatment of gout aims to lower the serum urate well below its solubility limit in tissue fluid [72, 73]. This leads to resorption of tophaceous material which will reduce and eventually prevent further attacks of gout. Ultrasound or MRI can now be used to monitor that such resorption is taking place.

\section{Conclusion}

In the authors' institution, the clinical practice of rheumatologists and radiologists varies amongst individuals. This is in the authors' view normal and reflects different training, experience and personal preference.

Between the specialties there are areas of mutual misunderstanding which are best resolved by a closer working relationship. Imaging can make a huge difference for long-term patient outcome. The pros and cons of using different imaging techniques are within the remit of radiologists (otherwise what is our point?). Both specialties have a duty to use imaging in a cost- 
effective manner to provide the requisite clinical information on diagnosis, prognosis and serial assessment with the least risk and inconvenience to the patient. This can be best achieved by close working relationships between the specialties.

Conflicts of interest The authors declare no conflicts of interest. No external funding was received for this study.

Open Access This article is distributed under the terms of the Creative Commons Attribution License which permits any use, distribution, and reproduction in any medium, provided the original author(s) and the source are credited.

\section{References}

1. Song IH, Heldmann F, Rudwaleit M et al (2011) Treatment of active ankylosing spondylitis with abatacept: an open-label, 24-week pilot study. Ann Rheum Dis 70(6):1108-1110

2. Sieper J (2012) How to define remission in ankylosing spondylitis? Ann Rheum Dis 71(Suppl 2):i93-i95

3. Haavardsholm EA, Boyesen P, Ostergaard M et al (2008) Magnetic resonance imaging findings in 84 patients with early rheumatoid arthritis: bone marrow oedema predicts erosive progression. Ann Rheum Dis 67(6):794-800

4. Narvaez JA, Narvaez J, De Lama E et al (2010) MR imaging of early rheumatoid arthritis. Radiographics 30(1):143-163, discussion 163-5

5. Jimenez-Boj E, Nobauer-Huhmann I, Hanslik-Schnabel B et al (2007) Bone erosions and bone marrow edema as defined by magnetic resonance imaging reflect true bone marrow inflammation in rheumatoid arthritis. Arthritis Rheum 56(4):1118-1124

6. McQueen FM, Ostendorf B (2006) What is MRI bone oedema in rheumatoid arthritis and why does it matter? Arthritis Res Ther 8(6):222

7. Dalbeth N, Smith T, Gray S et al (2009) Cellular characterisation of magnetic resonance imaging bone oedema in rheumatoid arthritis; implications for pathogenesis of erosive disease. Ann Rheum Dis 68(2): 279-282

8. Conaghan PG, O'Connor P, McGonagle D et al (2003) Elucidation of the relationship between synovitis and bone damage: a randomized magnetic resonance imaging study of individual joints in patients with early rheumatoid arthritis. Arthritis Rheum 48(1):64-71

9. Ostendorf B, Mattes-Gyorgy K, Reichelt DC et al (2010) Early detection of bony alterations in rheumatoid and erosive arthritis of finger joints with high-resolution single photon emission computed tomography, and differentiation between them. Skeletal Radiol 39(1):55-61

10. Wiles N, Lunt M, Barrett E et al (2001) Reduced disability at five years with ealry treatment of inflammatory polyarthritis: results form a large observational cohort, using propensity models to adjust for disease severity. Arthritis Rheum 44(5):1033-1042

11. Bukhari M, Wiles N, Lunt M et al (2003) Influence of diseasemodifying therapy on radiographic outcome in inflammatory polyarthritis at five years. Arthritis Rheum 48(1):46-53

12. Aletaha D, Neogi T, Silman AJ et al (2010) 2010 Rheumatoid arthritis classification criteria: an American College of Rheumatology/ European League Against Rheumatism collaborative initiative. Arthritis Rheum 62(9):2569-2581

13. Hermann KG, Backhaus M, Schneider U et al (2003) Rheumatoid arthritis of the shoulder joint: comparison of conventional radiography, ultrasound, and dynamic contrast-enhanced magnetic resonance imaging. Arthritis Rheum 48(12):3338-3349

14. Backhaus M, Burmester GR, Sandrock D et al (2002) Prospective two year follow up study comparing novel and conventional imaging procedures in patients with arthritic finger joints. Ann Rheum Dis 61(10):895-904

15. Lindegaard HM, Vallo J, Horslev-Petersen K et al (2006) Low-cost, low-field dedicated extremity magnetic resonance imaging in early rheumatoid arthritis: a 1-year follow-up study. Ann Rheum Dis 65(9): $1208-1212$

16. Boutry N, Larde A, Lapegue F et al (2003) Magnetic resonance imaging appearance of the hands and feet in patients with early rheumatoid arthritis. J Rheumatol 30(4):671-679

17. Winalski CS, Aliabadi P, Wright RJ et al (1993) Enhancement of joint fluid with intravenously administered gadopentetate dimeglumine: technique, rationale, and implications. Radiology 187(1):179-185

18. Conaghan P, Bird P, Ejbjerg B et al (2005) The EULAR-OMERACT rheumatoid arthritis MRI reference image atlas: the metacarpophalangeal joints. Ann Rheum Dis 64(Suppl 1):i11-i21

19. Ejbjerg B, McQueen F, Lassere M et al (2005) The EULAROMERACT rheumatoid arthritis MRI reference image atlas: the wrist joint. Ann Rheum Dis 64(Suppl 1):i23-i47

20. McQueen F, Ostergaard M, Peterfy C et al (2005) Pitfalls in scoring MR images of rheumatoid arthritis wrist and metacarpophalangeal joints. Ann Rheum Dis 64(Suppl 1):i48-i55

21. Weber U, Lambert RG, Ostergaard M et al (2010) The diagnostic utility of magnetic resonance imaging in spondylarthritis: an international multicenter evaluation of one hundred eighty-seven subjects. Arthritis Rheum 62(10):3048-3058

22. Baraliakos X, Hermann KG, Landewe R et al (2005) Assessment of acute spinal inflammation in patients with ankylosing spondylitis by magnetic resonance imaging: a comparison between contrast enhanced T1 and short tau inversion recovery (STIR) sequences. Ann Rheum Dis 64(8):1141-1144

23. Weber U, Ostergaard M, Lambert RG et al (2011) The impact of MRI on the clinical management of inflammatory arthritides. Skeletal Radiol 40(9):1153-1173

24. Ostergaard M, Peterfy C, Conaghan P et al (2003) OMERACT Rheumatoid Arthritis Magnetic Resonance Imaging Studies. Core set of MRI acquisitions, joint pathology definitions, and the OMERACT RA-MRI scoring system. J Rheumatol 30(6):1385-1386

25. Spencer SP, Ganeshalingam S, Kelly S et al (2012) The role of ultrasound in the diagnosis and follow-up of early inflammatory arthritis. Clin Radiol 67(1):15-23

26. Naredo E, Rodriguez M, Campos C et al (2008) Validity, reproducibility, and responsiveness of a twelve-joint simplified power doppler ultrasonographic assessment of joint inflammation in rheumatoid arthritis. Arthritis Rheum 59(4):515-522

27. Schueller-Weidekamm C (2009) Quantification of synovial and erosive changes in rheumatoid arthritis with ultrasound-revisited. Eur J Radiol 71(2):225-231

28. Platzgummer H, Schueller G, Grisar J et al (2009) Quantification of synovitis in rheumatoid arthritis: do we really need quantitative measurement of contrast-enhanced ultrasound? Eur J Radiol 71(2): 237-241

29. Szkudlarek M, Narvestad E, Klarlund M et al (2004) Ultrasonography of the metatarsophalangeal joints in rheumatoid arthritis: comparison with magnetic resonance imaging, conventional radiography, and clinical examination. Arthritis Rheum 50(7):2103-2112

30. Terslev L, Torp-Pedersen S, Savnik A et al (2003) Doppler ultrasound and magnetic resonance imaging of synovial inflammation of the hand in rheumatoid arthritis: a comparative study. Arthritis Rheum 48(9):2434-2441

31. Ozgul A, Yasar E, Arslan N et al (2009) The comparison of ultrasonographic and scintigraphic findings of early arthritis in revealing rheumatoid arthritis according to criteria of American College of Rheumatology. Rheumatol Int 29(7):765-768

32. Duer A, Ostergaard M, Horslev-Petersen K et al (2008) Magnetic resonance imaging and bone scintigraphy in the differential diagnosis of unclassified arthritis. Ann Rheum Dis 67(1):48-51 
33. Palosaari K, Vuotila J, Takalo R et al (2006) Bone oedema predicts erosive progression on wrist MRI in early RA - a 2-year observational MRI and NC scintigraphy study. Rheumatology (Oxford) 45(12): $1542-1548$

34. Roimicher L, Lopes FP, de Souza SA et al (2011) 99mTc-anti-TNF\{alpha\} scintigraphy in RA: a comparison pilot study with MRI and clinical examination. Rheumatology (Oxford) 50(11):2044-2050

35. Horger M, Schmalzing M, Ioanoviciu SD et al (2010) Ankylosing spondylitis: imaging findings in the axial skeleton. Rofo 182(8):639-643

36. Colbert RA (2010) Early axial spondyloarthritis. Curr Opin Rheumatol 22(5):603-607

37. Bozgeyik Z, Ozgocmen S, Kocakoc E (2008) Role of diffusionweighted MRI in the detection of early active sacroiliitis. AJR Am J Roentgenol 191(4):980-986

38. Madsen KB, Egund N, Jurik AG (2010) Grading of inflammatory disease activity in the sacroiliac joints with magnetic resonance imaging: comparison between short-tau inversion recovery and gadolinium contrast-enhanced sequences. J Rheumatol 37(2):393-400

39. Bredella MA, Steinbach LS, Morgan S et al (2006) MRI of the sacroiliac joints in patients with moderate to severe ankylosing spondylitis. AJR Am J Roentgenol 187(6):1420-1426

40. Bollow M, Fischer T, Reisshauer H et al (2000) Quantitative analyses of sacroiliac biopsies in spondyloarthropathies: $\mathrm{T}$ cells and macrophages predominate in early and active sacroiliitis- cellularity correlates with the degree of enhancement detected by magnetic resonance imaging. Ann Rheum Dis 59(2):135-140

41. Sieper J, Rudwaleit M, Baraliakos X et al (2009) The Assessment of SpondyloArthritis international Society (ASAS) handbook: a guide to assess spondyloarthritis. Ann Rheum Dis 68(Suppl 2):ii1-44

42. Gaspersic N, Sersa I, Jevtic V et al (2008) Monitoring ankylosing spondylitis therapy by dynamic contrast-enhanced and diffusionweighted magnetic resonance imaging. Skeletal Radiol 37(2):123-31

43. McGonagle D, Marzo-Ortega H, O'Connor P et al (2002) Histological assessment of the early enthesitis lesion in spondyloarthropathy. Ann Rheum Dis 61(6):534-537

44. Kotoura N, Sakamoto K, Fukuda Y et al (2011) Evaluation of magnetic resonance signal intensity in bone marrow after administration of super paramagnetic iron oxide (SPIO). Nihon Hoshasen Gijutsu Gakkai Zasshi 67(3):212-220

45. Blum A, Roch D, Loeuille D et al (2009) Bone marrow edema: definition, diagnostic value and prognostic value. J Radiol 90(12): 1789-1811

46. Bierry G, Jehl F, Boehm N et al (2009) Macrophage imaging by USPIO-enhanced MR for the differentiation of infectious osteomyelitis and aseptic vertebral inflammation. Eur Radiol 19(7):1604-1611

47. Maksymowych WP, Weber U (2011) Diagnostic utility of MRI in early spondyloarthritis. Curr Rheumatol Rep 13:402-408

48. Tins B, Cassar-Pullicino V, Haddaway M (2012) The 3d SPACE sequence for routine imaging of the spine-preliminary experience. Br J Radiol 85(1016):e480-e489

49. Weber U, Maksymowych WP, Jurik AG et al (2009) Validation of whole-body against conventional magnetic resonance imaging for scoring acute inflammatory lesions in the sacroiliac joints of patients with spondylarthritis. Arthritis Rheum 61(7):893-899

50. Rudwaleit M, Jurik AG, Hermann KG et al (2009) Defining active sacroiliitis on magnetic resonance imaging (MRI) for classification of axial spondyloarthritis: a consensual approach by the ASAS/ OMERACT MRI group. Ann Rheum Dis 68(10):1520-1527

51. Robinson PC, Wordsworth BP, Reveille JD et al (2013) Axial spondyloarthritis: a new disease entity, not necessarily early ankylosing spondylitis. Ann Rheum Dis 72(2):162-164

52. Madsen KB, Jurik AG (2010) MRI grading method for active and chronic spinal changes in spondyloarthritis. Clin Radiol 65(1):6-14

53. Boyesen P, McQueen FM, Gandjbakhch F et al (2011) The OMERACT Psoriatic Arthritis Magnetic Resonance Imaging Score
(PsAMRIS) is reliable and sensitive to change: results from an OMERACT workshop. J Rheumatol 38(9):2034-2038

54. National Institute for Health and Clinical Excellence (2008) Adalimumab, etanercept and infliximab for ankylosing spondylitis. NICE technology appraisal guidance 143. National Institute for Health and Clinical Excellence, London

55. Song IH, Carrasco-Fernandez J, Rudwaleit M et al (2008) The diagnostic value of scintigraphy in assessing sacroiliitis in ankylosing spondylitis: a systematic literature research. Ann Rheum Dis 67(11):1535-1540

56. Yildiz A, Gungor F, Tuncer T et al (2001) The evaluation of sacroiliitis using 99mTc-nanocolloid and 99mTc-MDP scintigraphy. Nucl Med Commun 22(7):785-794

57. Depasquale R, Kumar N, Lalam RK et al (2012) SAPHO: What radiologists should know. Clin Radiol 67(3):195-206

58. Colina M, Govoni M, Orzincolo C et al (2009) Clinical and radiologic evolution of synovitis, acne, pustulosis, hyperostosis, and osteitis syndrome: a single center study of a cohort of 71 subjects. Arthritis Rheum 61(6):813-821

59. Strobel K, Fischer DR, Tamborrini G et al (2010) 18F-fluoride PET/ CT for detection of sacroiliitis in ankylosing spondylitis. Eur J Nucl Med Mol Imaging 37(9):1760-1765

60. Rowbotham EL, Grainger AJ (2011) Rheumatoid arthritis: ultrasound versus MRI. AJR Am J Roentgenol 197(3):541-546

61. Mohammadi A, Ghasemi-Rad M, Aghdashi M et al (2013) Evaluation of disease activity in ankylosing spondylitis; diagnostic value of color Doppler ultrasonography. Skeletal Radiol 42:219-224

62. Spadaro A, Iagnocco A, Perrotta FM et al (2011) Clinical and ultrasonography assessment of peripheral enthesitis in ankylosing spondylitis. Rheumatology (Oxford) 50(11):2080-2086

63. Kiris A, Kaya A, Ozgocmen S et al (2006) Assessment of enthesitis in ankylosing spondylitis by power Doppler ultrasonography. Skeletal Radiol 35(7):522-8

64. D'Agostino MA, Said-Nahal R, Hacquard-Bouder C et al (2003) Assessment of peripheral enthesitis in the spondylarthropathies by ultrasonography combined with power Doppler: a cross-sectional study. Arthritis Rheum 48(2):523-533

65. Tinazzi I, McGonagle D, Biasi D et al (2011) Preliminary evidence that subclinical enthesopathy may predict psoriatic arthritis in patients with psoriasis. J Rheumatol 38(12):2691-2692

66. Aydin SZ, Karadag O, Filippucci E et al (2010) Monitoring Achilles enthesitis in ankylosing spondylitis during TNF-alpha antagonist therapy: an ultrasound study. Rheumatology (Oxford) 49(3):578-582

67. Chowalloor PV, Keen HI (2013) A systematic review of ultrasonography in gout and asymptomatic hyperuricaemia. Ann Rheum Dis 72(5):638-645

68. McQueen FM, Reeves Q, Dalbeth N (2013) New insights into an old disease: advanced imaging in the diagnosis and management of gout. Postgrad Med J 89(1048):87-93

69. Yu JS, Chung C, Recht M et al (1997) MR imaging of tophaceous gout. AJR Am J Roentgenol 168(2):523-527

70. Desai MA, Peterson JJ, Garner HW et al (2011) Clinical utility of dual-energy CT for evaluation of tophaceous gout. Radiographics 31(5):1365-1375, discussion 1376-7

71. Gerster JC, Landry M, Dufresne L et al (2002) Imaging of tophaceous gout: computed tomography provides specific images compared with magnetic resonance imaging and ultrasonography. Ann Rheum Dis 61(1):52-54

72. Zhang W, Doherty M, Bardin T et al (2006) EULAR evidence based recommendations for gout. Part II: Management. Report of a task force of the EULAR Standing Committee for International Clinical Studies Including Therapeutics (ESCISIT). Ann Rheum Dis 65(10):1312-1324

73. Zhang W, Doherty M, Pascual E et al (2006) EULAR evidence based recommendations for gout. Part I: Diagnosis. Report of a task force of the Standing Committee for International Clinical Studies Including Therapeutics (ESCISIT). Ann Rheum Dis 65(10):1301-1311 